\title{
"INFLUENCIA DE ALGUNS COMPOSTOS QUÍMICOS NA RETENÇÃO DO ÁCIDO ASCÓRBICO EM FRUTAS LIOFILIZADAS" *
}

\author{
HOMERO FONSECA ** \\ JoÃo NUNES NOGUEIRA ** \\ JORGE LEME JUNIOR $* * *$
}

\section{RESUMO}

\begin{abstract}
No presente trabalho foram estudados os efeitos do EDTA, do metabissulfito de potássio e de metabissulfito + ácido tartárico na retenção do ácido ascórbico durante a liofilização e posterior armazenamento de cereja das Antilhas e de morango.

Os resultados e as análises estatísticas mostraram que todos os tratamentos foram significativamente superiores às testemunhas, observandose também que o tratamento metabissulfito de potássio + ácido tartárico foi ligeiramente melhor que os demais tratamentos, porém não estatisticamente significativo.
\end{abstract}

\section{INTRODUÇÃO}

O emprego da liofilização como método de conservação de alimento é relativamente recente. $O$ processo apresenta a grande vantagem de desidratar o alimento a vácuo utilizando baixas temperaturas, resultando um produto de alta qualidade.

De um modo geral, os processos que as indústrias empregam para conservar os alimentos deixam muito a desejar, principalmente no que diz respeito à preservação de vitaminas. Estudando o teor de ácido ascórbico e beta-caroteno em frutas e hortaliças brasileiras, FONSECA \& NOGUEIRA (1968) e FONSECA et al. (1969) constataram este fato, destacando de modo especial, a perda de ácido ascórbico que ocorre durante a industrialização da goiaba.

\footnotetext{
* Agradecemos à FAPESP pelo liofilizador a nós cedido, à USAID pelo equipamento analítico e à ex-Cadeira de Horticultura da ESALQ pelas cerejas das Antilhas fornecidas. Entregue para publicação em 4-1-73.

** Depart.o de Tecnologia Rural.

*** Professor aposentado do Depart. ${ }^{\circ}$ de Tecnologia Rural.
} 
A estabilidade do ácido ascórbico durante o processo de liofilização tem sido objeto de muitas pesquisas. Assim, KALLISTRATOS \& SENGBUSCH (1964), KALLISTRATOS et al. (1965), HAMED \& FODA (1966) e CARBALLIDO et al. (1970) verificaram que a perda de ácido ascórbico era insignificante durante a liofilização. Entretanto, outros autores como TITOV et al. (1964), LEMPKA et al. (1966) e POPOVSKII \& IVASYUK (1968) afirmaram que, dependendo do alimento, pode ocorrer uma perda de 10 a $50 \%$ da vitamina durante o citado processo. Por outro lado GINNETTE \& KAUFMAN (1968) e PORDAB et al. (1971) citaram que, dependendo das condições de armazenamento, podem também ocorrer perdas apreciáveis de ácido ascórbico no produto desidratado.

Segundo SANTAGOSTINO \& SAPETTI (1963), a estabilidade do ácido ascórbico está condicionada à presença de algumas substâncias, como é o caso do $\mathrm{SO}_{2}$ que tem a capacidade de retardar a sua oxidação. Em 1965, FUKUDA (1965) realizou várias experiências procurando testar a estabilidade do ácido ascórbico em presença de alguns compostos químicos. Verificou êle que o cloreto de sódio, ácido cítrico, peptona, glicina e dehidroacetato de sódio tinham a capacidade de estabilizar o ácido ascórbico, porém, na ausência de cobre. FUKUDA et al. (1966 a) chegaram à mesma conclusão utilizando alginato de sódio. Entretanto, na presença de cobre, apenas este último se mostrou eficiente (FUKUDA et al, 1966 b).

DRAUDT \& HUANG (1966), trabalhando com frutas liofilizadas constataram a eficiência do $\mathrm{SO}_{2}$ na retenção de ácido ascórbico, principalmente, em condições mínimas de umidade. Em estudo semelhante, FERRIOLS et al. (1968) verificaram que o $\mathrm{SO}_{2}$ além de aumentar a retenção do ácido ascórbico, melhorou as propriedades organoléticas e permitiu empregar temperaturas mais elevadas, diminuindo assim o tempo de desidratação do produto.

Tendo em vista estes fatos, os autores se propuseram, neste trabalho, a estudar a influência de alguns compostos químicos na retenção do ácido ascórbico durante a liofilização e armazenamento do produto desidratado, em cereja das Antilhas e morango, frutas consideradas como ótimas fontes daquela vitamina.

\section{MATERIAL E MÉTODOS}

\section{Material}

A cereja das Antilhas (Malpighia punicifolia L.) utilizada neste trabalho foi coletada no pomar da Escola Superior de Agricultura "Luiz de Queiroz" e o morango (Flagaria vesca L.) foi adquirido no mercado local, ambas em estado de pleno amadurecimento, tendo sido utilizadas apenas as partes comestíveis das mesmas. 


\section{Métodos}

a) Preparo das polpas (purê) para liofilização

O purê das polpas foi obtido da seguinte maneira:

Cereja das Antilhas: por esmagamento contra peneiras de malhas de $2 \mathrm{~mm}$, ficando separadas as sementes.

Tratamento : foi imediatamente adicionado à polpa, o sal sódico do EDTA, dissolvido na menor quantidade de água, de maneira a permitir uma concentração de $300 \mathrm{ppm}$ no purê, e a seguir liofilizados.

Morango: retirados o pedúnculo e as sépalas e desintegrado em liquidificador. Imediatamente antes da desintegração os morangos foram submetidos a dois tipos de tratamentos sendo, a seguir, liofilizados.

Tratamento 1: imersão em solução de metabissulfato de potássio a $1 \%$, durante 30 minutos.

Tratamento 2 : imersão em solução contendo $1 \%$ de metabissulfito de potássio, mais $5 \%$ de ácido tartárico, durante 30 minutos.

\section{b) Liofilização}

A liofilização dos purês foi feita logo em seguida, em um liofilizador SULENE, modelo único. Os purês foram congelados a $-40^{\circ} \mathrm{C}$ e, a seguir feito o vácuo. Durante a liofilização a pressão absoluta na câmara de desidratação foi mantida em $0,1 \mathrm{~mm}$ de mercúrio e a temperatura do condensador foi de aproximadamente $-60^{\circ} \mathrm{C}$. O calor para sublimação foi suprido através de resistências elétricas contidas no interior das três prateleiras e controladas, por meio de termostatos, para permanecerem à temperatura de $40^{\circ} \mathrm{C}$. A marcha da desidratação foi controlada por termômetros (pares térmicos) colocados no seio do purê. Durante a desidratação, a temperatura do purê permaneceu em torno de $-20^{\circ} \mathrm{C}$. Após a elevação da temperatura, sua permanência durante duas horas a $40^{\circ} \mathrm{C}$, foi considerada como sendo o final da desidratação. $O$ vácuo no liofilizador foi quebrado com introdução do gás nitrogênio. Os pós resultantes foram colocados em frascos de vidro âmbar de $100 \mathrm{ml}$, fechados com tampas plásticas, parafinados e colocados em caixas de papelão ao abrigo da luz.

Para a cereja das Antilhas foi feita apenas uma liofilização e para o morango foram feitas duas, identificadas por morango I e II. 


\section{c) Análises químicas}

As análises de ácido ascórbico foram efetuadas: a) na fruta fresca (purê), b) imediatamente após a liofilização e c) a intervalos de um mês, por períodos variáveis, a saber:

cereja das Antilhas - 10 meses
morango I $\quad-2$ meses
morango II -3 meses

O método utilizado para dosagem do ácido ascórbico foi o fotocolorimétrico de ORSINI \& PAULA SANTOS (1943), modificado por LEME JR. \& MALAVOLTA (1950) e que se baseia na reação entre o ácido ascórbico e o reativo de Tillmans. $\mathrm{O}$ colorímetro usado foi o Coleman Junior, modelo $6 \mathrm{D}$ e comprimento de onda $540 \mathrm{~nm}$.

A umidade foi determinada por diferença de peso da amostra seca em estufa a $110^{\circ} \mathrm{C}$, sob vácuo de $60 \mathrm{~mm}$ de mercúrio, até peso constante.

A determinação do Brix foi efetuada em refratômetro manual Bauch \& Lomb, modelo GD 8861, (0-60\%).

Todas as determinações foram feitas em duplicata.

\section{d) Análise estatística}

Para a análise estatística dos resultados foi feita a análise da variância, com utilização do teste F (GOMES, 1970). A comparação das médias foi feita pelo teste Tukey, sendo este aplicado somente nos casos em que os valores de $F$ eram significativos ao nível de 5 ou $1 \%$ de probabilidade (não foi calculado para o morango, pois o experimento teve pouca duração). Foi também calculado o coeficiente de variação (C.V.).

\section{RESULTADOS E DISCUSSÃO}

Os resultados das análises químicas constam dos QUADROS $1 \mathrm{e}$ 2 e representam a média das determinações. Os resultados da análise estatística constam dos QUADROS 3 e 4.

Como se pode observar todos os tratamentos apresentaram resultados positivos. Com relação à cereja das Antilhas (ver QUADRO 1) o tratamento com EDTA foi bastante eficiente na retenção do ácido ascórbico, principalmente durante o processo de desidratação. Nesta fase a perda da vitamina no material tratado não chegou a 7\%, nível este bastante satisfatório em comparação com a testemunha que sofreu uma perda duas vezes e meia maior. Embora na literatura pesquisada não tenha sido encontrada nenhuma citação 
QUADRO 1. - Influência do tratamento com EDTA no teor de ácido ascórbico em cereja das Antilhas liofilizada.

\begin{tabular}{|c|c|c|}
\hline $\begin{array}{c}\text { Epoca da } \\
\text { análise }\end{array}$ & $\begin{array}{c}\text { Testemunha } \\
\text { mg/100g }\end{array}$ & $\begin{array}{l}\text { Tratado } \\
\mathrm{mg} / 100 \mathrm{~g}\end{array}$ \\
\hline Polpa fresca* & 695 & 695 \\
\hline Matéria seca $* *$ & 8338 & 8440 \\
\hline Pós-liofilização *** & 6921 & 7767 \\
\hline $1 .^{\circ}$ mês & 5600 & 6485 \\
\hline 2.0 mês & 5671 & 6196 \\
\hline 3.0 mês & 5351 & 6140 \\
\hline $4 .^{\circ}$ mês & 5351 & 6123 \\
\hline $5 .^{\circ}$ mês & 5335 & 6123 \\
\hline $6 .^{\circ}$ mês & 5101 & 5848 \\
\hline $7 .^{\circ}$ mês & 4575 & 5560 \\
\hline 8..$^{\circ}$ mês & 4300 & 5370 \\
\hline $9 .^{\circ}$ mês & 4065 & 5150 \\
\hline 10.0 mês & 4000 & 5100 \\
\hline Perda no processo & $17 \%$ & $6,8 \%$ \\
\hline Perda no armazenamento & $42 \%$ & $34 \%$ \\
\hline Perda mensal média & $4,2 \%$ & $3,4 \%$ \\
\hline
\end{tabular}

* Brix $-8,0$

* Resultados que seriam obtidos se não houvesse perda durante a liofilização *** Umidade $-4,0 \%$ 
QUADRO 2. - Influência do tratamento com metabissulfito de potássio e ácido tartárico no teor de ácido ascórbico em morango liofilizado.

\begin{tabular}{|c|c|c|c|c|c|c|}
\hline \multirow{2}{*}{$\begin{array}{l}\text { Época da } \\
\text { Análise }\end{array}$} & \multicolumn{3}{|c|}{$\begin{array}{c}\text { Morango I } \\
\mathrm{mg} / 100 \mathrm{~g}\end{array}$} & & \multicolumn{2}{|c|}{$\begin{array}{l}\text { Morango II } \\
\mathrm{mg} / 100 \mathrm{~g}\end{array}$} \\
\hline & Test. & Trat. 1 & Trat. 2 & Test. & Trat. 1 & Trat. 2 \\
\hline Polpa fresca * & 55 & 55 & 55 & 37 & 49 & 51 \\
\hline Matéria seca ** & 753 & 753 & 753 & 709 & 708 & 700 \\
\hline Pós-liofilização *** & 440 & 716 & 728 & 449 & 588 & 601 \\
\hline $1 .^{\circ}$ mês & 430 & 711 & 725 & 435 & 577 & 595 \\
\hline $2 . .^{\circ}$ mês & 413 & 700 & 718 & 428 & 571 & 590 \\
\hline 3.0 mês & - & - & - & 420 & 565 & 586 \\
\hline Perda no processo & $41,6 \%$ & $5 \%$ & $3,4 \%$ & $36,7 \%$ & $17 \%$ & $14 \%$ \\
\hline $\begin{array}{l}\text { Perda no armaze- } \\
\text { namento }\end{array}$ & $6 \%$ & $2,2 \%$ & $1,3 \%$ & $6 \%$ & $4 \%$ & $2,4 \%$ \\
\hline $\begin{array}{l}\text { Perda mensal } \\
\text { média }\end{array}$ & $3 \%$ & $1,1 \%$ & $0,6 \%$ & $2 \%$ & $1,3 \%$ & $0,8 \%$ \\
\hline
\end{tabular}

* Brix - Morango I : 7,0

Morango II: 5,0

* Resultados que seriam obtidos se não houvesse perda durante a liofilização

*** Umidade - 4,0\%

QUADRO 3. - Análise estatística dos resultados da cereja das Antilhas liofilizada

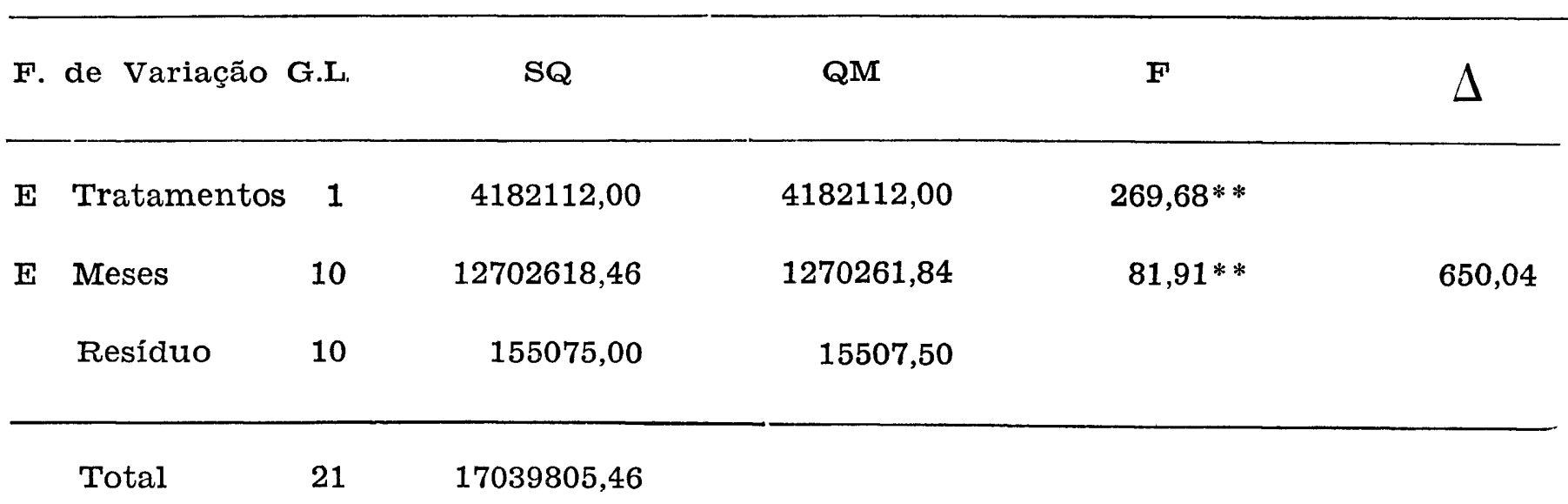

* Significativo ao nível de $1 \%$ de probabilidade - C. V. $=2,2 \%$ 
QUADRO 4. - Análise estatística dos resultados do morango liofilizado

\begin{tabular}{|c|c|c|c|c|c|}
\hline $\mathbf{F}$. & de Variação & G.L. & $\mathbf{S Q}$ & QM & $\mathbf{F}$ \\
\hline $\mathrm{E}$ & Repetições & 1 & 32930,04 & 32930,04 & $17,40 * *$ \\
\hline \multirow[t]{3}{*}{$\mathbf{E}$} & Tratamentos & 11 & 296083,46 & 26916,67 & $14,22 * *$ \\
\hline & Resíduo & 11 & 20817,46 & 1892,49 & \\
\hline & Total & 23 & 349830,96 & & C.V. $=7,0 \%$ \\
\hline & E Testemunha & 3 & 134229,38 & 44743,12 & $23,64 * *$ \\
\hline & Tratamento & 1 & 11539,00 & 3846,33 & 2,03 \\
\hline & Tratamento & 2 & 6844,50 & 2281,50 & 1,21 \\
\hline
\end{tabular}

C.V. $=3,8 \%$

* Significativo ao nível de $1 \%$ de probabilidade.

com relação ao emprego do EDTA, o resultado obtido era de se esperar pois, segundo SANTAGOSTINO \& SAPETTI (1963) a estabilidade do ácido ascórbico está condicionada à presença de substâncias que sejam capazes de retardar a sua oxidação. Outros autores como TITOV et al. (1964), LEMPKA et al. (1966) e POPOVSKII \& IVASYUK (1968) afirmaram que, dependendo do alimento, podem ocorrer perdas de até $50 \%$ da vitamina durante a liofilização, se nenhum tratamento preventivo for aplicado. A perda verificada durante o armazenamento pode ser considerada razoável em se tratando de uma substância facilmente oxidável e considerando-se não só que o produto foi armazenado à temperatura ambiente como também que o tempo de armazenamento foi relativamente longo. Realmente tudo indica que, dependendo das condições de armazenamento, podem ocorrer perdas apreciáveis de ácido ascórbico no produto desidratado (GINNETTE \& KAUFMAN, 1968 e PORDAB et al., 1971). Durante o período citado a perda no material tratado foi $8 \%$ menor do que a perda sofrida pela testemunha, sendo a perda mensal média de 3,4 e $4,2 \%$, respectivamente.

A análise estatística dos resultados demonstrou existir uma diferença altamente significativa tanto entre os tratamentos (material tratado e não tratado) como também entre épocas de análises (meses). 
Para o morango (ver QUADRO 2) a combinação de metabissulfito de potássio com ácido tartárico (Trat. 2) revelou possuir grande eficiência na retenção do ácido ascórbico no produto liofilizado. Quando utilizado sozinho, o metabissulfito de potássio (Trat. 1) apresentou também uma eficiência bastante razoável. A perda de ácido ascórbico pela testemunha, durante a desidratação, foi bastante elevada (41,6 e 36,7\% respectivamente, nas liofilizações morango I e II) em comparação com o Trat. 1 (5 e 17\%) e Trat. 2 (3,4 e14\%). A conclusões semelhantes chegaram também SANTAGOSTINO \& SAPETTI (1963), FUKUDA et al. (1966) e DRAUDT \& HUANG (1966). FERRIOLS et al. (1968) verificaram que o $\mathrm{SO}_{2}$ além de aumentar a retenção do ácido ascórbico ainda melhorou as propriedades organoléticas do produto desidratado. Durante o armazenamento pôde-se observar também que a perda total da vitamina no material tratado foi insignificante, principalmente em Morango I (2,2\% no Trat. $1 \mathrm{e}$ $1,3 \%$ no Trat. 2). Do mesmo modo a perda mensal média do mesmo material foi igualmente insignificante. Por razões que desconhecemos os tratamentos utilizados foram bem mais eficientes em Morango I do que em II, pois em ambos os casos foram utilizadas as mesmas condições de processamento, acondicionamento e armazenamento.

A análise estatística dos resultados demonstrou existir uma diferença altamente significativa entre os tratamentos (testemunha, Trat. 1 e Trat. 2). Para os Trats. 1 e 2 não houve diferença estatística significativa entre épocas de análise (meses), porém para a testemunha foi constatada uma diferença estatística altamente significativa. Este fato vem demonstrar mais uma vez a eficiência dos tratamentos utilizados.

\section{CONCLUSÕES}

Desta maneira conclui-se que:

a) os três tratamentos utilizados para proteger o ácido ascórbico, contra a oxidação, foram eficientes.

b) dentre os tratamentos, a associação de metabissulfito de potássio com ácido tartárico produziu efeitos ligeiramente superiores aos demais.

c) novas pesquisas deverão ser efetuadas com aqueles tratamentos abordando também o aspecto das propriedades organoléticas dos produtos tratados, antes que se possa recomendar sua aplicação generalizada. 


\section{SUMMARY}

INFLUENCE OF SOME CHEMICAL COMPOUNDS ON THE RETENTION OF ASCORBIC ACID IN FREEZE-DRIED FRUITS.

In the present work the effects of EDTA, potassium metabisulfite and potassium metabisulfite + tartaric acid were studied in the retention of ascorbic acid during freeze-drying and storage of West Indies cherry (Malpighia punicifolia (L.) and of strawberry (Flagaria vesca $L$.).

The results and the statistical analysis showed that all treatments were significantly superior to the control. It was also observed that potassium metabisulfite + tartaric acid was slightly better than the other treatments but not statistically significant.

\section{LITERATURA CITADA}

CARBALLIDO, A., L. RUBIO \& J. MARIA, 1970. - Use of lyophilization for the preservation of strawberries. An. Bromatol., 22:229-54.

DRAUDT, H. N. \& I. Y. HUANG, 1966 - Effect of moisture content of freeze-dried peaches and bananas on changes during storage related to oxidative and carbonylamine browning. J. Agr. Food Chem., 14:170-76.

FERRIOLS, B. L., J. V. CARBONELL \& F. PINAGA, 1968 - Effect of drying conditions on the quality of freeze-dried green beans. Rev. Agroquim. Tecnol. Aliment., 8:371-80.

FONSECA, H. \& J. N. NOGUEIRA, 1968 - Conteúdo de ácido ascórbico em produtos industrializados de goiaba. Arq. Bras. Nutrição, 24:135-39.

FONSECA, H., J. N. NOGUEIRA \& A. M. S. MARCONDES, 1969 - Teor de ácido ascórbico e beta-caroteno em frutas e hortaliças brasileiras. Arch. Lat. Nutricion, 19:9-16.

FUKUDA, T., 1965 - Stabilities of ascorbic acid, ascorbate, erythorbic acid and sodium erythorbate solutions coexisting in foods. I. Using redistilled water. Showa Yakka Daigaku Kiyo, 3:91-99.

FUKUDA, T., M. ISHIBASHI \& A. KOSUKA, 1966 a - Effect of food additives on the stability of ascorbic acid and sodium ascorbate solutions. I. Use of redistilled water. Showa Yakka Daigaku Kiyo, 4:35-42.

FUKUDA, T., K. MIYAKAWA \& K. RO, $1966 \mathrm{~b}$ - Effect of food additives on stability of ascorbic acid and sodium ascorbate solutions. III. Sodium alginate. Shokuhin Eiseigaku Za Zasshi, 7:508-13.

GINNETTE, L. F. \& V. F. KAUFMAN, 1968 - Freeze-drying of foods. In: TRESSLER, D. K., W. B. V. ARSDEL \& M. J. COPLEY, eds. - The Freezing Preservation of Foods, vol. 3, The Avi. Publ. Co. p. 377-403.

GOMES, F. P., 1970 - Curso de Estatística Experimental, 4.. ed., Piracicaba, E.S.A, "Luiz de Queiroz" p. 29-41. 
HAMED, M. G. E. \& Y. H. FODA, 1966 - Freeze-drying of onions. Z. Lebensm. Untersuch. - Forch., 130:220-27.

KALLISTRATOS, G. \& R. SENGBUSCH, 1964 - Comparison of the losses in various food components under freeze-drying and other drying methods. Nutr. Dieta, 6:193-202.

KALLISTRATOS, G., E. RICHTER \& R. SENGBUSCH, 1965 - Grundlagen für die industrielle verwendung der gefriertrocknung zur "Konservierung" von obst und gemuse. Die Industrielle obst-und Gemüseverwertung, 5, marz.

LEME Jr., J. \& E. MALAVOLTA, 1950 - Determinação fotométrica do ácido ascórbico. Anais da E.S.A. "Luiz de Queiroz", Univ. de S. Paulo, 7:116-129.

LEMPKA, A., W. PROMINSKI \& J. SULKOWSKA, 1966 - Losses of L-ascorbic acid during lyophilization of selected berries. Pr. Zakresu Towarozn. Chem., Wysza Szk. Ekon. Poznaniu Zesz. Nauk., Ser. I N.0 26, 23-27.

ORSINI, D. \& O. PAULA SANTOS, 1943 - Determinação da vitamina C em alguns frutos brasileiros pelo colorímetro fotoelétrico. Separata da Resenha Clínico-Científica, S. Paulo. Ano XII (12).

POPOVSKII, V. G. \& N. T. IVASYUK, 1968 - Chemicotechnological investigation of fruits and berries for sublimation drying. Tr. Mold. Nauch. Issled Inst. Pishch. Prom. 8:40-51.

PORDAB, Z., J. PIECHANOWSKI \& L. MAIK, 1971 - Powdered vegetable-cereal and fruit-cereal purees for children. II. Losses of L-ascorbic acid and B-carotene and the shelf life of the powdered purees during storage. Przem-Spozyw, 21:109-18.

SANTAGOSTINO, E. A. \& C. SAPETTI, 1963 - Ascorbic acid oxidation in the presence and absence of $\mathrm{SO}_{2}$. Ann. Sper. Agrar., 17:561-81.

TITOV, N. N., T. P. PETROVSKAYA \& T. N. SAKHAROVA, 1964 - The vitamin $\mathrm{C}$ content in products dried by sublimation. Sb Tr. Leningr. Inst. Soc. Torgovli, N.' 23, 95-99. 

